\title{
Management of the innovative ecosystem development environment
}

\author{
Olga Kolomytseva ${ }^{1}$, Inna Gunina ${ }^{2, *}$,Elena Kolesnikova ${ }^{1}$ and Vera Agafonova ${ }^{3}$ \\ ${ }^{1}$ Voronezh State University of Engineering Technologies, 19, Revolution Av., Voronezh, 394036, \\ Russia \\ ${ }^{2}$ Voronezh State Technical University, 14, Moscow Av., Voronezh, 394026, Russia \\ ${ }^{3}$ Moscow State University of Civil Engineering, 26, Yaroslavskoye shosse, Moscow, 129337, Russia
}

\begin{abstract}
One of the key factors of innovative development is the availability of a favorable innovative environment ensuring the transformation of ideas and developments into market products, introduction of these products into most important branches of economics and social sphere, as well as allowing saving the unique set of scientific and engineering schools. But the key problem for implementation of effective innovations is the absence of favorable environment and innovative climate promoting creation of innovations, ensuring the growth of global competitive ability, labor productivity, and life quality of population. Thus, the formation and development of the innovative environment as the most important condition for implementation of effective innovations represents the actual scientific task of significant national economic importance. Digital technologies are best developed in a harmonious ecosystem. Nowadays, ecosystem-based projects are becoming more popular, when a community of people connected by common interests is formed; the economy is very rapidly migrating into the history of ecosystems. In the next 10 years, we should expect the competition of ecosystems, digital platforms integrated between each other: in the upcoming global digital economy, that economic contour will win, which will be the fastest growing in the number and quality of digital platforms integrated into a single ecosystem. That is, the digital economy should be seen as an ecosystem economy.
\end{abstract}

\section{Introduction}

The "environment" forms the conditions for the existence of an object or process, the manifestation of their essence, and can show itself both in its relative stability and in changes corresponding to the essence of the object. In the environment, there are changes in the form of object or process, and it has an active impact on them. Thus, the "environment" is set of conditions which ensuring the stable existence of object or process. Unlike to the "environment" and "sphere", the "space" has complex structure and large scale of coverage, and "global space" is mediated by civilizational measures [1].

\footnotetext{
*Corresponding author: 642663@mail.ru
} 
Innovative environment can be considered from two points of view: as the development environment and as the interaction environment. The development environment forms and conducts the changes that are necessary to achieve a new sustainability of the socioeconomic system as a whole, with the preservation, accumulation and transformation of the necessary qualities. The interaction environment ensures the interaction between all elements and components directly or indirectly included into innovative environment [2].

The innovative system is, firstly, part of the innovation environment, and, secondly, the innovative system is incorporated into the national socio-economic system and the global external environment (i.e. the innovative system is considered by us as subsystem of socioeconomic environment or as an element that forms the structure of the latter). The innovative system ensures functioning and sets impetus for searching the directions of stability development for all economic agents and for the society as a whole. The innovative environment carries out transport function, transmitting innovations produced by the innovative system to the level of socio-economic systems and global environment [3].

\section{Materials and Methods}

Taking into account the modern realities and tendencies, the innovative environment should be considered at the following levels:

- level L1: mega- (global level);

- level L2: meso-level 1 (mega-macro);

- level L3: macro- (national level of country, state);

- level L4: meso-level 2 (macro-micro);

- level L5: micro- (enterprise level).

The levels of the innovative environment are distinguished by the subjects and objects of innovation activity that are located on them, the degree of localization and scale. Structurally and functionally coordinated, they form the basic and intermediate (meso-) levels of vertical and horizontal interactions. Meso-spaces serve as intermediate layers and at the same time act as generators of changes in the states of the basic levels of the innovative environment [4].

BCG experts have developed the innovative development pyramid, or shortly - the pyramid of innovations, in order to structure the concept of innovative environment. From the authors' point of view, in the conditions of digitalization, the pyramid of the BCG innovative environment should be updated using the matrix of the National Technological Initiative (NTI) - a key element of the NTI, which determines the logic of interaction between all its participants [5].

There are six layers in the center of attention of actualized innovative pyramid:

1) institutions: basic conditions for public life;

2) knowledge (talents): conditions for appearance of researches;

3) culture: conditions for development;

4) technologies, platforms and services: directions for development;

5) infrastructure: conditions for activity;

6) market: commercialization.

An important indicator is the degree of sustainability of pyramid, which can be determined due to parameters variations indexes (institutes, knowledge, culture, technologies, infrastructure, markets) within frameworks of each innovative environment. Thus, for target state of the innovative environment, the innovative pyramid must be balanced [6].

The essence of the innovative environment formation is the reaching of global competitive ability and outstripping development of socio-economic system, maximization of labor productivity, whereof it results that cross-border effective interaction of business, 
scientific and educational community and state, development of high-technology businesses in traditional and high-technology markets, increase of competitive ability in global market should be considered as the main aspect of goal-setting of the innovative environment formation at all levels. Under conditions of fourth industrial revolution, the set of locomotive branches and connected with it technologies and scientific foundations is changing. Technologies aimed not at the production and distribution of goods and services, but at the person himself are of key importance. These are biotechnologies, new medicine, robotics, nanotechnology, cognitive technologies, High-Hume technologies, new use of natural resources, etc. All of this is much more focused on the human than technological priorities of the previous structure. The core of the sixth technological structure may become NBIC-convergence. NBIC-technologies (Nano-Bio-Info-Cogno-technologies) are understood as breakthrough innovative technologies in the field of convergence of nanotechnologies, ICT, biotechnologies, and cognitive technologies. Many modern new technologies are located at the intersection of NBIC group, for example, construction of cellular and tissue structures (nano+bio), new informational interfaces (info+cogno), bioinformatics (nano+info+bio) [7].

Forming an innovative environment of socio-economic systems can be considered effective if it means obtaining global, national and local effects in the form of global leadership in high-tech markets, maximizing productivity and quality of life, the number of people employed in the innovation and digital economy, the place of the Russian Federation in Global Innovation Index, as well as if as a result of the formation of the innovative environment, the effect of self-organization of the innovative system and synergy are observed.

\section{Results}

One of the most significant research elements being core element of methodology and allowing implementing the concept of the innovative environment formation is a concept model.

First unit reflects the current state of the innovative environment of socio-economic system characterizing with current level of global competitiveness, labor efficiency, life quality, and number of persons engaged in the innovative economics. These characteristics determine the innovative environment level. The essence of this unit is the determination of goal-setting of the innovative environment formation process [5]. The foregoing reasoning and conceptual foundations of the innovative environment formation allow determining the goal-setting as:

- Maximizing the level of global leadership (competitiveness) in high-technology markets $\mathrm{L}_{\mathrm{gl}} \rightarrow \max$

- Maximizing the labor efficiency LE $\rightarrow$ max;

- Increase in the life quality of population LQ $\rightarrow$ max;

- Increase in the number of persons engaged in the innovative system NEIS $\rightarrow$ max.

In addition, it is worth noting that the availability as target criteria $\mathrm{L}_{\mathrm{gl}} \rightarrow \max , \mathrm{LE} \rightarrow$ $\max$, LQ $\rightarrow$ max, NEIS $\rightarrow$ max supposes the necessity of their quantitative measurement.

Second unit of conceptual model represents the process of formation and development of the innovative environment. In accordance with the proposed concept [6], the objects, functions and levels of formation of the innovative environment are taken as vectors of decomposition.

First decomposition vector allows detailing objects of organizational and managing impacts in accordance with the ground components of the innovative environment. In the model under consideration, the following are singled out as objects of the formation of the innovation environment: institutions, knowledge (talents), culture, technology, services, 
infrastructure, markets. Second decomposition vector reflects the main functions of the innovative environment formation. Among these functions, the authors consistently distinguished: forecasting, strategy and planning, coordination, implementation, monitoring and control. Third decomposition vector allows taking into account the differences and subordination of goals and tasks of the environment formation during the process of the innovative environment formation, as well as differences in objects and managing forms at mega-level, meso-level 1 (mega-macro), macrolevel, meso-level 2 (macro-micro), microlevel [8].

Thus, the process of formation and development of the innovative environment ensures the considered above principles of sustainable development, integration, and complexity:

- Principle of sustainable development is carried out due to sustainable innovative environment formation at all levels (management horizons): $\mathrm{L}=\mathrm{L} 1 \cup \mathrm{L} 2 \cup \mathrm{L} 3 \cup \mathrm{L} 4 \cup \mathrm{L}$;

- Implementation of the principle of integration is carried out due to mutual penetration of subsystems of the innovative environment, i.e. $0=01 \cup 02 \cup 03 \cup 04 \cup 05$;

- Innovative environment formation in context of managing functions ensures the implementation of the principle of complexity in favor of combination of five functions: $\mathrm{F}=\mathrm{F} 1 \cup \mathrm{F} 2 \cup \mathrm{F} 3 \cup \mathrm{F} 4 \cup \mathrm{F} 5$.

The last, third unit of the conceptual model is associated with the reflection of the result of the formation of the innovative environment and the fixation of the resulting state of the socio-economic system after the implementation of the processes of the environment formation.

From the standpoint of the first block of the conceptual model, the results of the formation of the innovative environment can be written as [9]:

$$
\left\{\begin{array}{c}
\mathrm{L}_{\mathrm{gl}} \rightarrow \max \\
\mathrm{LE} \rightarrow \max \\
\mathrm{LQ} \rightarrow \max \\
\mathrm{NEIS} \rightarrow \max
\end{array}\right.
$$

The achievement in the third unit of the goals established in the first unit ensures the targeted formation and development of the innovative environment of the socio-economic system.

\section{Discussions}

Digital ecosystem is an independent group of acting subjects (people, technical tools and organizations) sharing standardized digital platforms to interact with each other to achieve a commercial or social goal. A digital ecosystem is a set of interconnected digital tools and technologies integrated into the business processes of an economic system.

Front-line creation of innovations is ensured by the innovative ecosystem, which represents the interaction environment ensuring the communication between all elements and components, directly or indirectly included into the innovative environment. The innovative environment, in its turn, is a broader concept than the innovative ecosystem, and includes the development environment as well - it forms and implements the changes that are necessary to achieve a new sustainability of the socio-economic system as a whole with preservation, accumulation and transformation of necessary qualities. Hence, a narrower understanding of the innovative environment through innovative ecosystem makes it possible to use such terms as "ecosystem operator", "services ecosystem", internet-platform as ecosystem and others.

Creation of the innovative ecosystem can be considered as a target state of the innovative environment transformation. The innovative environment transformation, its specificity and principles of implementation considered in this research, in its theoretical, 
methodological and empirical essence assume that the socio-economic system as evolving and developing object needs the special managing mechanism.

The transformation of the innovative environment of socio-economic systems should be considered as the condition for interchanging or changing the way of functioning and development of these systems, based on practical implementation of knowledge and ensures the turnover of phases of sustainability (equilibrium) and not sustainability of the mentioned systems. The turnover of phases of the state of socio-economic systems means the acquisition (accumulation) by the system of new qualities, as well as their preservation and furthering transformation necessary for ensuring of sustained life activity of this system. It is worth noting that modern transformation allows ensuring not only adaptability of the development of innovative environment, but first gives possibility to the innovative environment to be counter- adaptive, be able to ensure advance internal changes in favor of proactive control (control aimed at forecasting changes in the future).

As an initial thesis, when developing the mechanism for transforming an innovative environment, we take the following: conditions for the micro level in the innovation system are created at the macro level, and the micro level generates the innovations required for the development of the innovative environment as a whole. In the transformation of the innovative environment, we consider it preferable to use a system-synergetic methodological approach, which is a constructive tool to describe and model the transformation of the innovative environment of socio-economic systems [10].

Taking into account the foregoing, we consider that the concept of innovative environment transformation must be formed with the use of systematic and synergic approaches and be aimed at creation of tools for eliminating the barriers in the innovative environment.

For the formation of the transformation mechanism of the innovative environment, the emergent behavior of the system components is of interest. Through dynamic interaction, the components of a specific complex system can develop characteristics or patterns that are not directly expressed in them. The transformation of the innovative environment must be built into the managed system, so that the management mechanisms are consistent with the mechanisms and patterns of self-organization and self-regulation [11].

One of the organizational methods of the transformation mechanism of the innovative environment in the conditions of digitalization is the adoption of at least some of the components of the methodology of agile development, also known as agile.

Agile-transformation is the organization of activities by clearly setting goals and allocating the necessary resources to achieve them, creating work teams with a high degree of autonomy and using digital technologies to improve the efficiency and coordination of employees' work [12]. A distinctive feature of the agile organization is its ability to quickly respond to environmental changes in the conditions of high uncertainty.

\section{Conclusion}

The intensive development of a digital economy society inevitably entails challenges in knowledge management. Priority resources are information and knowledge gained from expert experience. And competencies, being competitive advantages in the market, are innovativeness, competence, creativity, cognition. Therefore, the transition from physical to digital economy requires fundamentally new approaches not only in industries and productions, but also in the areas of formation of personnel potential for the digital economy: education, training, formation and management of innovative human capital. 


\section{References}

1. N.V. Rudakova, Development of tools of ensuring of competitive ability of socialeconomics systems for implementation of strategies of innovative development. Part 2, Collectively monograph (2012)

2. A.N. Maloletko, Contemporary Problems of Social Work 2-4, 58-66 (2016)

3. T. Tolstykh, E. Shkarupeta, Y. Kostuhin, A. Zhaglovskaya, Proceedings of the 31th International Business Information Management Association Conference (IBIMA) (Milan, 2018)

4. I. Karapetyants, Y. Kostuhin, T. Tolstykh, E. Shkarupeta, A. Krasnikova, Proceedings of the 30th International Business Information Management Association Conference (IBIMA) (Madrid, 2017)

5. I. Karapetyants, Y. Kostuhin, T. Tolstykh, E. Shkarupeta, E. Syshsikova, Proceedings of the 30th International Business Information Management Association Conference (IBIMA) (Madrid, 2017)

6. S. Vasin, L. Gamidullaeva, E. Shkarupeta, A. Finogeev, I. Palatkin, European Research Studies Journal XXI-3, 63-76 (2018)

7. B. Walrave, M. Talmar, K.S. Podoynitsyna, A.G.L. Romme, G.P.J. Verbong, Technological Forecasting and Social Change (2017) ISSN 0040-1625, https://doi.org/10.1016/j.techfore.2017.04.011

8. A. Radziwon, M. Bogers, Technological Forecasting and Social Change (2018) ISSN 0040-1625, https://doi.org/10.1016/j.techfore.2018.04.021

9. M. Holgersson, O. Granstrand, M. Bogers, Long Range Planning 51-2, 303-319 (2018) ISSN 0024-6301, https://doi.org/10.1016/j.lrp.2017.08.007

10. T. Tolstykh, E. Shkarupeta, Y. Kostuhin, A. Zhaglovskaya, Proceedings of the 31th International Business Information Management Association Conference (IBIMA), 4738-4746 (2018)

11. T. Tolstykh, Y. Vertakova, E. Shkarupeta, I. Shishkin, K. Krivyakin, Proceedings of the 29th International Business Information Management Association Conference (IBIMA), 2180 - 2191 (2017)

12. T. Tolstykh, E. Shkarupeta, I. Shishkin, O. Dudareva, N. Golub, Advances in Intelligent Systems and Computing 622, 736-743 (2018) DOI: 10.1007/978-3-31975383-6 